\title{
Segregación de Mn en películas delgadas de GaAsMn obtenidas mediante pulverización catódica
}

\section{Segregation of Mn into GaAsMn thin films prepared by magnetron sputtering}

\author{
José Doria-Andrade ${ }^{1,2}$, Camilo Pulzara-Mora ${ }^{1}$, \\ Roberto Bernal-Correa ${ }^{3}$, Andrés Rosales-Rivera ${ }^{4}$, \\ Álvaro Pulzara-Mora ${ }^{1}$
}

\footnotetext{
${ }^{1}$ Laboratorio de Nanoestructuras Semiconductoras, Grupo Magnetismo y Materiales Avanzados, Universidad Nacional de Colombia, Manizales, Caldas, Colombia.

${ }^{2}$ Laboratorio de Materialografia, Facultad de Ingeniería, Institución Universitaria Pascual Bravo, Medellín, Antioquia, Colombia.

${ }^{3}$ Unidad de Docencia y Formación, Universidad Nacional de Colombia, Sede Orinoquia, Kilometro 9 vía Arauca-Caño Limón, Arauca, Casanare, Colombia.

${ }^{4}$ Laboratorio de Magnetismo y Materiales Avanzados, Universidad Nacional de Colombia, Sede Manizales, Manizales, Caldas, Colombia.

e-mail: j.doriaan@pascualbravo.edu.co,cpulzaram@unal.edu.co rabernalco@unal.edu.co, arosalsesr@unal.edu.co, aopulzram@unal.edu.co
}

\section{RESUMEN}

Actualmente, la fabricación de películas delgadas de GaAs dopadas con Mn (GaAsMn) sobre Si (100), es un objeto de gran interés debido a su posible integración con la tecnología del silicio, generando un desarrollo significativo en la funcionalidad de los dispositivos optoelectrónicos y espintrónicos. En este trabajo, presentamos un estudio sistemático de la caracterización estructural, morfológica, óptica, y magnética de películas delgadas de GaAsMn preparadas por pulverización catódica R.F sobre un substrato de silicio (100), para temperaturas del crecimiento de 100 y $200{ }^{\circ} \mathrm{C}$, respectivamente. A partir de los espectros Raman se identificaron los modos vibracionales, trasversal óptico (TO) y longitudinal óptico (LO) de GaAs, localizados en $290 \mathrm{~cm}^{-1}$ y $265 \mathrm{~cm}^{-1}$, respectivamente. Adicionalmente, se identificaron modos vibracionales de MnAs, debido a la substitucion de átomos de Ga por átomos de Mn en altas concentraciones. La segregación de Mn, fue corroborada mediante difracción de rayos-X, en donde se evidencian planos cristalinos en las direcciones (400) y (200) de GaAs policristalino, y planos cristalográficos pertenecientes a fases de $\mathrm{Mn}_{1+\mathrm{x}} \mathrm{As}$. La morfología y el modo de crecimiento de las películas delgadas de GaAsMn/Si (100), se llevó a cabo mediante imágenes de microscopía de fuerza atómica (AFM) y microscopía electrónica de barrido (SEM) tomadas sobre la superficie y en sección transversal, respectivamente. Finalmente, un análisis de las propiedades magnéticas de las películas delgadas de GaAsMn a partir de imágenes de microscopía de fuerza magnética (MFM), revelan la presencia de dominios magnéticos superficiales provenientes de MnAs. Concluimos que las propiedades físicas de las películas de GaAsMn dependen de las condiciones de crecimiento.

Palabras-clave: Magnetrón Sputtering r.f, Semiconductores Magnéticos Diluidos, GaAsMn.

\section{ABSTRACT}

Currently, the manufacture of thin films of GaAs doped with Mn (GaAsMn) on Si (100), is an object of great interest, due to its possible integration with silicon technology, generating a significant development in the functionality of the devices optoelectronics and spintronics. In this work, we present a systematic study of the structural, optical, morphological and magnetic characterization of GaAsMn thin films prepared by R.F sputtering on a $\mathrm{Si}(100)$ substrate, for growth temperatures of 100 and $200 \mathrm{oC}$, respectively. From Raman spectra, the transverse optical (TO) and longitudinal optical (LO) vibrational modes, located at $290 \mathrm{~cm}^{-1}$ and $265 \mathrm{~cm}^{-1}$ of GaAs were identified. Additionally, vibrational modes of MnAs were identified, due to the replacement of 
$\mathrm{Ga}$ atoms with $\mathrm{Mn}$ atoms in high concentrations. The segregation of Mn was bear out by X-ray diffraction, where crystalline planes are evidenced in the directions (400) and (200) of polycrystalline GaAs, and crystallographic planes belonging to the $\mathrm{Mn}_{1+\mathrm{x}} \mathrm{As}$ phases. The morphology and growth mode of the thin films of GaAsMn / Si (100), was carried out by means of atomic force microscopy (AFM) and scanning electron microscopy (SEM) images, taken on the surface and in cross-section, respectively. Finally, an analysis of the magnetic properties of the GaAs thin films made from magnetic force microscopy (MFM) images, evidence surface magnetic domains formation, which depending on growth conditions. We concluded that, all properties of GaAsMn thin films are depending on growth conditions.

Keywords: Magnetron Sputtering r.f, Diluted Magnetic Semiconductors, GaAsMn.

\section{INTRODUCCIÒN}

El estudio de semiconductores III-V como: GaAs, InGaAs, GaAsN, InGaP ha sido objeto de interés en los últimos años debido a la versatilidad de sus propiedades ópticas y eléctricas, para aplicaciones en el desarrollo de dispositivos optoelectrónicos y en la tecnología fotovoltaica [1, 2, 3, 4, 5, 6]. Con el fin de incrementar el espectro de aplicaciones y generar reducción de los costos de producción, la investigación reciente se centra en la obtención de este tipo de aleaciones semiconductoras utilizando nuevas estrategias originando una importante actividad en el desarrollo y estudio de nuevos materiales [7, 8]. Un ejemplo de interés, es el aprovechamiento de materiales que combinan propiedades eléctricas (carga) y magnéticas (espín), como es el caso de los llamados semiconductores magnéticos diluidos (SMD), con los cuales se han alcanzado significativos avances a nivel de laboratorio para el desarrollo de dispositivos espintrónicos de tercera generación [9, $10,11,12]$.

En los semiconductores magnéticos diluidos, cuando la matriz de semiconductores III-V se dopa con metales de transición, ej. Mn o Cr, el espín de los electrones puede usarse como un grado de libertad adicional [13], generando distintos comportamientos: Magnético, cuando la componente del operador de spin promedio a lo largo del campo, $\langle\mathrm{Sz}\rangle$ es diferente de cero en la presencia de campo magnético externo y en el caso contrario y en ausencia de un campo magnético, se comporten como un semiconductor. En ese sentido, su desarrollo se convierte en la clave para el perfeccionamiento de dispositivos de spin, en donde la corriente de electrones "espín polarizados", puede modificar la dirección de magnetización de un electrodo ferromagnético [14], dando lugar a diversas aplicaciones en la creación de nuevos dispositivos espintrónicos, como válvulas de espín y dispositivos de almacenamiento de información.

La preparación de semiconductores magnéticos diluidos (DMS) III-V-Mn (tema de interés en el presente trabajo), se ha realizado mediante distintas técnicas epitaxiales $[15,16,17,18]$. Sin embargo, el alto costo que implica la implementación de la mayoría de estas técnicas a nivel industrial genera la exploración de métodos alternativos que permitan obtener películas delgadas de buena calidad estructural a un menor costo de producción $[19,20]$. En este sentido, en el presente trabajo nos enfocamos en el estudio estructural, composicional, óptico y magnético de películas delgadas películas delgadas de GaAsMn preparadas por pulverización catódica asistida por campo magnético, con el fin de correlacionar los parámetros experimentales con las propiedades físicas de estas aleaciones, y valorar la pertinencia de la técnica para la obtención de aleaciones III-V: Mn.

\section{MATERIALES Y METODOS}

Con el fin de identificar efectos asociados a la incorporación de átomos de Mn en la estructura de GaAs, se prepararon películas delgadas de GaAs dopadas con Mn por pulverización catódica R.F sobre sustratos de $\mathrm{Si}$ (100), a bajas temperaturas del substrato. Previo a la deposición de las películas, los sustratos fueron sometidos previamente a un tratamiento de limpieza en una cubeta ultrasónica durante 10 minutos en alcohol y acetona con el fin de remover la grasa superficial. Las películas delgadas se depositaron por pulverización simultánea de blancos comerciales (99.99) de GaAs ( 2 pulgadas) y Mn (1 pulgada) de alta pureza a potencias de las fuentes r.f de $30 \mathrm{Watt}$ y $15 \mathrm{Watt}$, respectivamente. Las películas se depositaron a temperaturas de sustrato de $100^{\circ} \mathrm{C}(\mathrm{M} 1)$ y $200^{\circ} \mathrm{C}(\mathrm{M} 2)$. Otras condiciones experimentales de la preparación de las películas se muestran en la tabla 1.

La caracterización estructural de las películas se realizó mediante de difracción de rayos-X (PANalytical Xpert Pro), equipado con una fuente de radiación de $\mathrm{Cu} \mathrm{K} \alpha(\lambda=1,540562 \AA)$ a una velocidad de $1 \%$ min, en un rango de $20^{\circ} \leq 2 \theta \leq 60^{\circ}$, con el fin de evitar la reflexión principal del substrato y maximizar la reflexión de la película. Las medidas Ráman se realizaron utilizando una configuración de retrodispersión con dirección perpendicular a la muestra (espectrómetro Ráman Dilor XY LabRAM equipado con un microsco- 
pio Olympus BX40 y una láser de longitud de onda de $632 \mathrm{~nm}$ con una potencia de $20 \mathrm{~mW}$ ), con el fin de determinar las frecuencias de los modos vibracionales TO y LO de GaAs y los modos asociados a la formación de clusters de MnAs. Para determinar la morfología y estimar el espesor de las películas, se tomaron imágenes de microscopia electrónica de barrido (FE-SEM), en un equipo JEOL JSM740 1F. Un estudio morfológico y superficial, se realizó utilizando microscopía de fuerza atómica (AFM) con un microscopio SPM marca Nanosurf modelo Easyscan2. Finalmente, utilizamos microscopía de fuerza magnética (MFM) para la distribución espacial de los dominios magnéticos de las películas de GaAsMn.

Tabla 1: Condiciones experimentales de la preparación de las películas de GaAsMn.

\begin{tabular}{l|c}
\hline \multicolumn{1}{c|}{ Parámetros } & Muestras (M1, M2) \\
\hline Presión residual (Torr) & $1.0 \times 10^{-6}$ \\
Presión de trabajo (mTorr) & $10 \times 10^{-3}$ \\
Distancia blanco - sustrato (cm) & 5 \\
Gas de trabajo & Argón \\
Temperatura de crecimiento $\left({ }^{\circ} \mathrm{C}\right)$ & $100(\mathrm{M} 1), 200(\mathrm{M} 2)$ \\
Potencia fuente r.f (Watt) & 40 \\
Tiempo de crecimiento (min) & 60 \\
\hline
\end{tabular}

\section{RESULTADOS}

\subsection{Rayos-X}

La Figura 1 muestra los resultados de difracción de rayos-x de las películas delgadas obtenidas a temperaturas de $100^{\circ} \mathrm{C}(\mathrm{M} 1)$ y $200^{\circ} \mathrm{C}(\mathrm{M} 2)$, respectivamente. En los difractogramas se observan planos cristalográficos (200), (220), (311) y (400) de GaAs. Adicionalmente, se identifican los planos cristalográficos (111) y (202) pertenecientes a $\mathrm{Mn}_{2}$ As y el plano (224) de la fase $\gamma$ de óxido de manganeso $\left(\gamma-\mathrm{Mn}_{2} \mathrm{O}_{3}\right)$ [21], debido a oxidación de la superficie por exposición con el aire. Al comparar los resultados de M1) y M2) no se observa un cambio significativo en las intensidades de los picos correspondientes a GaAs, pero la posición de los planos de GaAs presentan un corrimiento en $2 \theta=2^{\circ}$, debido a esfuerzos de aleación. Algunos reportes indican que las fases asociadas a $\mathrm{Mn}_{2} \mathrm{As}$ y $\gamma-\mathrm{Mn}_{2} \mathrm{O}_{3}$ pueden desaparecer realizando un tratamiento térmico a las muestras a una temperatura aproximada de $840^{\circ} \mathrm{C}$.

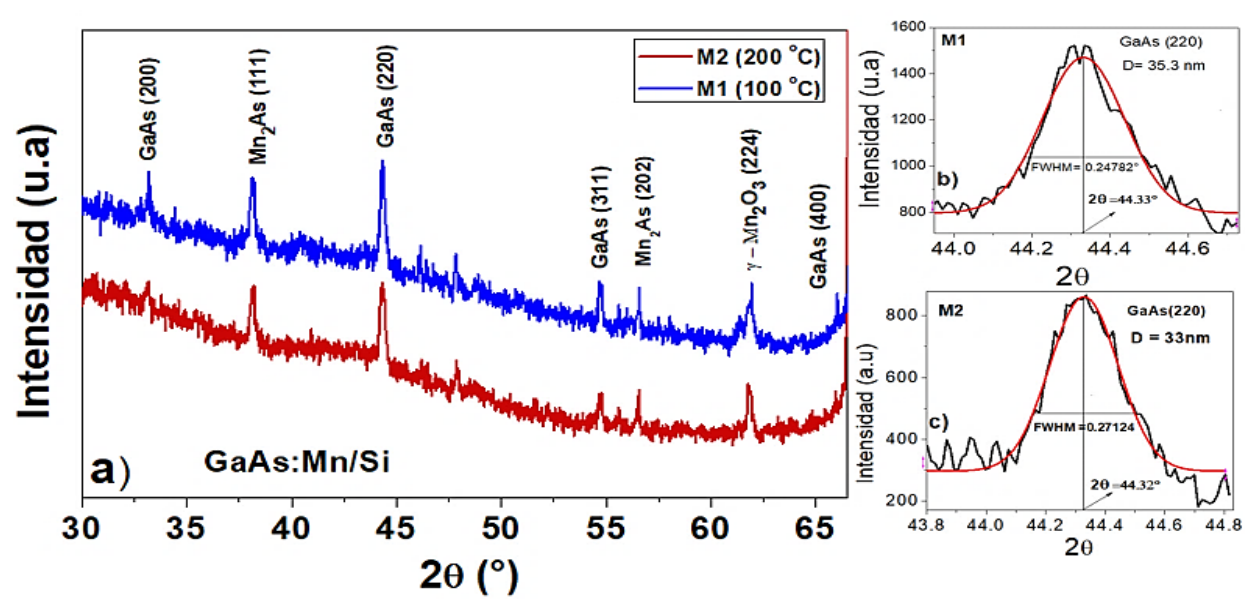

Figura 1: a) Resultados de difracción de rayos - X de películas delgadas de GaAsMn depositadas sobre silicio a diferentes temperaturas del substrato. En el lado derecho se muestran las figuras b) y c) con un ajuste de una función Gaussiana del plano (220) para M1) y M2), respectivamente.

A partir de los resultados de rayos-X, se realizó un estimativo del tamaño de cristalito utilizando la fórmula de Scherrer [22]. Para tal efecto se realizó una deconvolución del pico (200) de GaAs utilizando una Gaussiana que permitió determinar el ancho medio a la mitad del pico y el ángulo de difracción, como se 
indica en el lado derecho de la Fig. 1.

$$
D=\frac{k \lambda}{\beta \cos \theta}
$$

donde $\beta$ es el ancho medio del pico (FWHM), $\lambda$ es la longitud de onda de los rayos-X $\left(\mathrm{Cu}-\mathrm{K}_{\alpha}=0.154 \mathrm{~nm}\right), \mathrm{k}$ $=0.9$ es una constante relacionada con la geometría del cristalito y $\theta$ es el ángulo de difracción y $\mathrm{D}$ es el tamaño de cristal. Los valores obtenidos de tamaño de cristalito (D) fueron de $35.3 \mathrm{~nm}$ y $33.0 \mathrm{~nm}$ para las muestras M1) y M2), respectivamente.

Un estimativo del valor del parámetro de red de las muestras M1) y M2) se realizó, tomando en cuenta los planos $\left(\mathrm{h}_{1} \mathrm{k}_{1} \mathrm{l}_{1}\right)=(220)$ y $\left(\mathrm{h}_{2} \mathrm{k}_{2} \mathrm{l}_{2}\right)=(331)$, utilizando las ecuaciones (2) para una estructura cubica Zinc blenda:

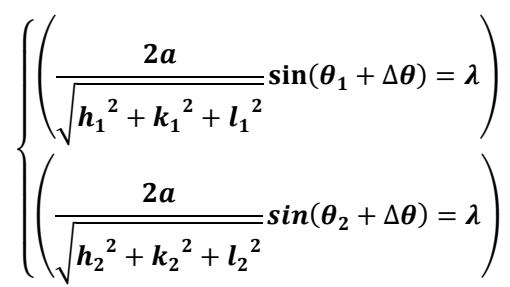

donde $\lambda$ es la longitud de onda de los rayos X. Resolviendo para $\Delta \theta$ en la ecuación (2), se obtiene

$$
a=\left(\frac{2 \lambda}{\sin \left(\theta_{2}+\Delta \theta\right)}\right) \quad \text { ó } \quad a=\left(\frac{\lambda}{\sin \left(\theta_{1}+\Delta \theta\right)}\right)
$$

El valor estimado usando la ecuación (2) de $\Delta \theta=4.976522^{\circ}$. Obteniendo un valor de $\mathrm{a}_{\mathrm{GaAs}}=0.575790$ $\mathrm{nm}$ para M1) y $\mathrm{a}_{\mathrm{GaAs}}=0.575790 \mathrm{~nm}$ para M2), que son comparables con el valor el reportado en la literatura [23].

\subsection{Espectroscopía Raman}

Considerando la alta sensibilidad de espectroscopia Ráman para discriminar modos vibracionales (fonones) asociados a impurezas y/o defectos en la estructura cristalina del semiconductor y con el fin de determinar la homogeneidad de las películas, se tomaron espectros Raman en distintos puntos de las muestras. En la figura 2 se muestran los espectros obtenidos para las películas delgadas depositadas a temperaturas de $100{ }^{\circ} \mathrm{C}(\mathrm{M} 1)$ y $200{ }^{\circ} \mathrm{C}$ (M2), donde se evidencian los modos vibracionales longitudinal óptico (LO) y transversal óptico (TO) de GaAs. Se observa, además, un corrimiento de $5 \mathrm{~cm}^{-1}$ en la posición de los modos vibracionales TO y LO con el aumento de la temperatura, lo que puede estar asociado con esfuerzos inducidos al momento de preparación debido al desacople de los parámetros de red y por efectos térmicos del sustrato y de la película [24]. Adicionalmente, se observan el modo $2 \mathrm{TO}(\Gamma)$ de GaAs situado en $532 \mathrm{~cm}^{-1}$. Resultados similares han sido reportados en capas de GaN dopadas con $\mathrm{Mn}$ [25].

Un modo plasmon acoplado al modo LO (CLOPM: Coupled LO-phonon plasmon mode), en $269 \mathrm{~cm}^{-1}$ se observa entre los modos TO y $\mathrm{LO}$ el cual se debe a la presencia de portadores libres, debido a fluctuaciones en el potencial del cristal. Se ha reportado que, tanto los modos TO, LO y el modo CLOPM se corren hacia bajas energías con el aumento del contenido de Mn [26]. Los modos transversal acústicos TA(X), 2TA(X) y DALA(X), localizados en $75.4 \mathrm{~cm}^{-1}, 153.7 \mathrm{~cm}^{-1}$ y $195 \mathrm{~cm}^{-1}$ respectivamente, provienen de vibraciones activadas por desorden estructural. [27]. Resultados similares se han reportado en muestras de GaAs por la implantación de iones de Mn en un cristal de GaAs [28]. 


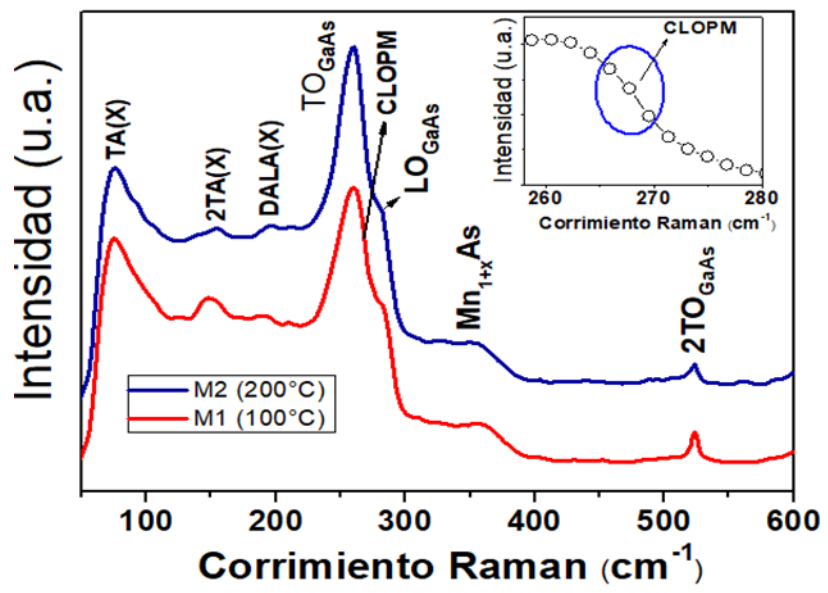

Figura 2: Espectros Raman de películas delgadas de GaAsMn depositadas sobre silicio, utilizando una línea láser de longitud de onda de $632 \mathrm{~nm}$ como fuente de excitación. En el inset, se muestra la frecuencia Raman del modo CLOPM.

\subsection{Microscopía Electrónica de Barrido y Microanálisis Elemental (SEM - EDS)}

La Fig. 4 a) muestra una imagen SEM tomada en sección transversal de la muestra M1) a 33.000X aumentos. En la imagen se observa una interfaz muy bien definida entre la película y el sustrato, evidenciando un crecimiento uniforme y columnar a lo largo del espesor de la película, típico del crecimiento por pulverización catódica. El espesor de las películas es de aproximadamente $500 \mathrm{~nm}$. En la figura $3 \mathrm{~b}$ ) se muestra una micrografía de la capa M1) tomada sobre la superficie, donde se observa una superficie plana con una baja rugosidad $\left(\mathrm{R}_{\mathrm{RMS}} 1.62 \mathrm{~nm}\right)$, compuesta de nanopartículas con tamaño del orden de $30 \mathrm{~nm}$ de diámetro. Sobre la superficie se observa inclusiones de Mn, debido probablemente a la segregación asociada a las fases mencionadas en los resultados de rayos X. Una imagen similar se obtuvo en la película M2).
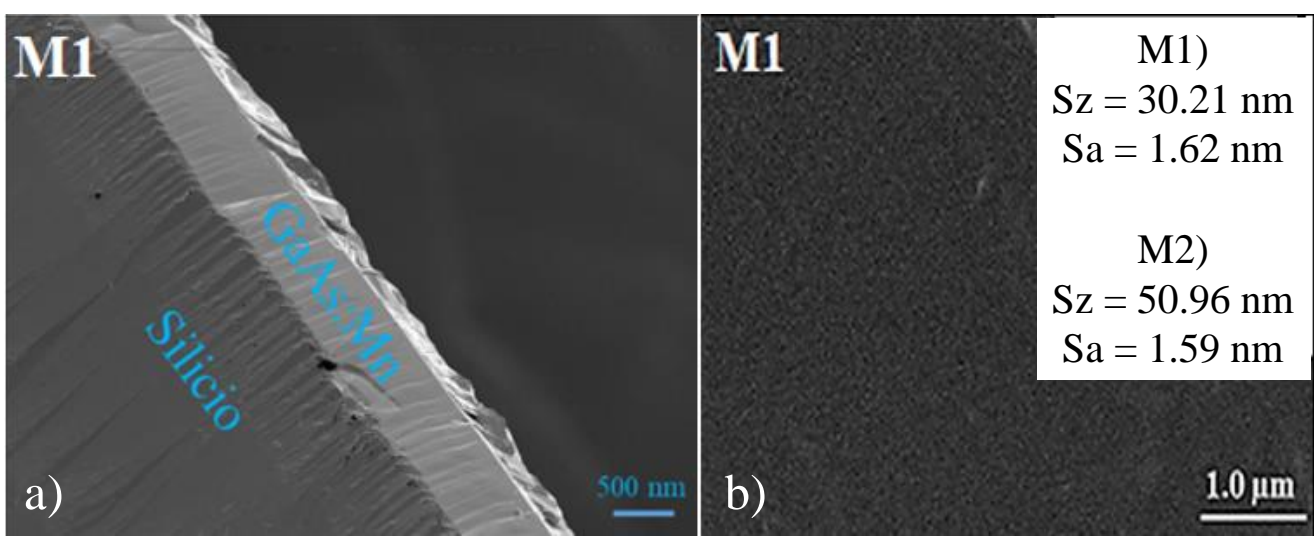

Figura 3: a) Imagen SEM en sección transversal a 33.000X aumentos para la película M1). b) Imagen SEM para la capa M1), tomada sobre la superficie.

Con el fin de corroborar la presencia de Mn en las películas de GaAsMn se realizó un microanálisis elemental SEM-EDS, utilizando espectrometría de rayos - X de energía dispersa (EDS). Para cada muestra se tomaron 5 espectros en diferentes regiones en un barrido de $5 \times 2.5 \mu \mathrm{m}^{2}$, obteniendo valores en $\%$ atómico sin variaciones significativas para los diferentes elementos, lo que indica una alta homogeneidad en composición en las películas de GaAsMn. En la figura 4 se muestra el espectro EDS obtenido para M1) en el área indicada en el recuadro. En el espectro se identificaron señales asociadas a la línea $K \alpha$ de Ga, As y Mn, respectivamente. Los resultados obtenidos del promedio de cada elemento se muestran en la tabla 2. 


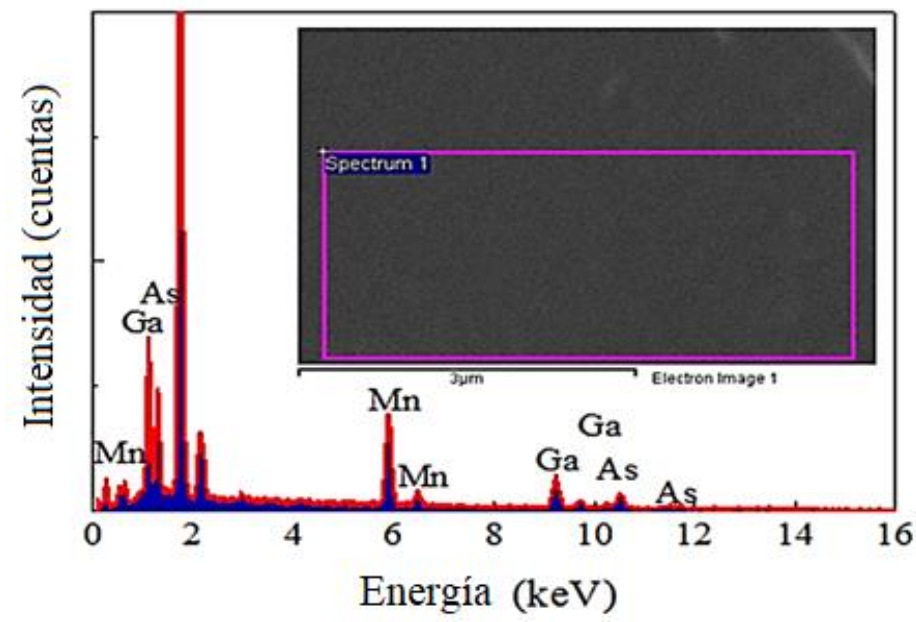

Figura 4: Espectro de EDS para la muestra M1). En el recuadro se muestra la imagen SEM en una escala de $3 \mu \mathrm{m}$, en donde se tomó el espectro EDS.

Tabla 2: Condiciones experimentales de la preparación de las capas de GaAsMn

\begin{tabular}{c|c|c}
\hline ELEMENTO & PESO\% & ATÓMICO\% \\
\hline \multicolumn{3}{|c}{ M1) } \\
\hline Mn & 31.82 & 38.05 \\
Ga & 33.31 & 31.38 \\
As & 34.87 & 30.57 \\
\hline Mn & M2) \\
Ga & 27.79 & 33.62 \\
As & 35.26 & 33.61 \\
\end{tabular}

\subsection{Microscopía de Fuerza Atómica (AFM)}

Un estudio morfológico complementario se realizó mediante microscopia de fuerza atómica (AFM). En la Fig. 5 se muestran las imágenes tomadas en un área de $3 \times 3 \mu \mathrm{m}^{2}$, para las películas delgadas M1) y M2). En los dos casos se observa una superficie altamente homogénea, lo cual fue corroborado desde los valores promedio de rugosidad y tamaño de grano. En la imagen de la película crecida a $100{ }^{\circ} \mathrm{C}(\mathrm{M} 1)$ se observan algunas partículas dispersas sobre una superficie plana probablemente asociados a regiones ricas en $\mathrm{Mn}$, debido a una menor movilidad de los átomos de Mn sobre el sustrato como una consecuencia de la baja temperatura de crecimiento, generando segregación de fases de GaAs y $\mathrm{Mn}_{1+\mathrm{x}} \mathrm{As}$, como se observa en los resultados de rayos - X. Un resultado similar se obtuvo para muestra crecida a una temperatura de $200{ }^{\circ} \mathrm{C}(\mathrm{M} 2)$, lo cual está de acuerdo con lo descrito en secciones anteriores. La no evidencia de la formación de granos en las imágenes AFM, se puede explicar por la menor área seleccionada para la toma de imágenes.

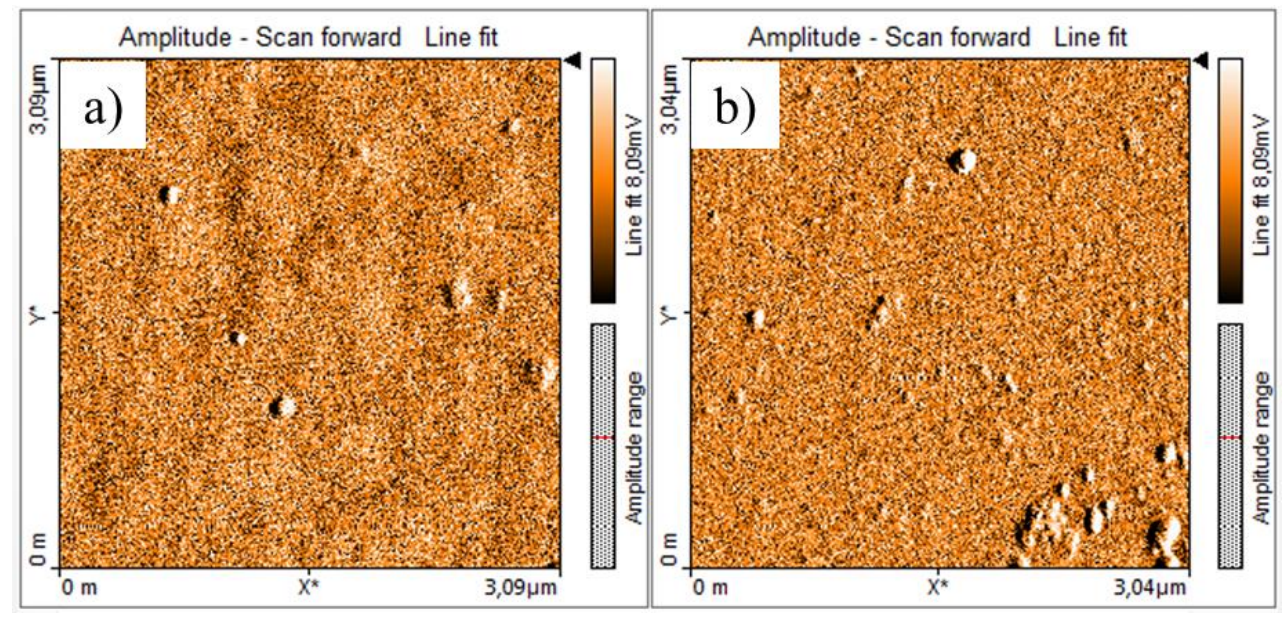


Figura 5: Imágenes de AFM tomadas en una region de $3 \times 3 \mu \mathrm{m}^{2}$ en las películas delgadas de GaAsMn crecidas a temperaturas de a) $100{ }^{\circ} \mathrm{C}$ y b) $200{ }^{\circ} \mathrm{C}$, muestras M1) y M2) respectivamante.

\subsection{Microscopía de Fuerza Magnética (MFM)}

Las imágenes que se muestran en la Fig. 6, corresponden medida de MFM tomadas a temperatura ambiente (300 K) en las muestras M1) y M2) sobre un barrido de $3 \times 3 \mu \mathrm{m}^{2}$. La formación de aglomeraciones (clusters) de MnAs en la matriz de GaAs presenta propiedades magnéticas interesantes que, dependen de la temperatura de preparación de las películas. Las imágenes de MFM permiten, identificar la distribución espacial de los dominios magnéticos sobre la superficie de la película, a partir de los cambios en la frecuencia de resonancia del cantiléver inducido por la dependencia del campo magnético entre la punta de la sonda magnética y la película. Las regiones más brillantes marcan áreas más sensibles al comportamiento paramagnético, mientras que las áreas más oscuras corresponden a dominios ferromagnéticos, lo cual significa que se pueden utilizar para almacenar información magnética en estas aleaciones semi-magnéticas.
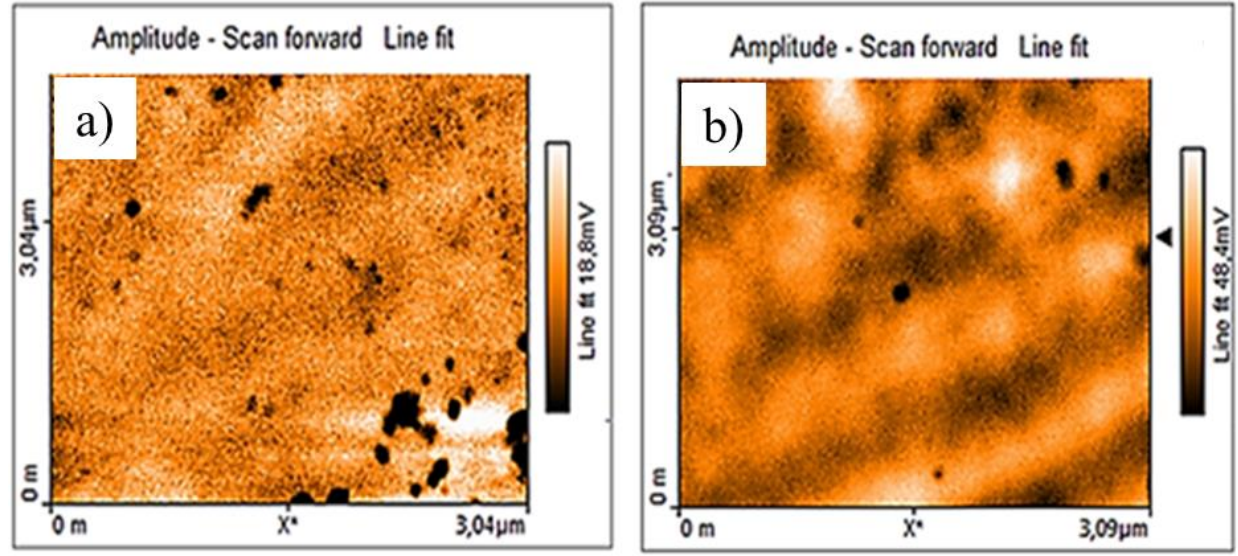

Figura 6: Imágenes de MFM en un área de $3 \times 3 \mu \mathrm{m}^{2}$ sobre la superficie de las películas delgadas de GaAsMn preparadas a temperatura del sustrato de: a) $100{ }^{\circ} \mathrm{C}$ y b) $200{ }^{\circ} \mathrm{C}$, correspondientes a las muestras M1) y M2).

Se ha reportado que por encima $125^{\circ} \mathrm{C}$ la estructura ortorrómbica se relaja y el material experimenta una transición de fase de segundo orden (puramente estructural) y vuelve a su estructura hexagonal original, mostrando propiedades paramagnéticas ( $\gamma$-fase) [29]. La formación de la fase MnAs ortorrómbica está de acuerdo con los resultados Raman discutidos (Fig. 3) Las figuras 6 muestra el contraste topográfico de las muestras M1) y M2), respectivamente, en donde se observan En regiones brillantes y oscuras, lo que indica, posiblemente, a la presencia de dominios magnéticos con diferentes orientaciones magnéticas. Este contraste en las imágenes de MFM se debe posiblemente a la presencia de la fase $\alpha$-MnAs (crestas) que corresponden a la componente ferromagnética, débil en este caso, mientras que los valles a la componente paramagnética $\beta$ MnAs.

\section{CONCLUSIONES}

Con los resultados obtenidos en este trabajo, se logró demostrar que la selección adecuada de parámetros experimentales (ejemplo: temperatura), favorece la cinética de los iones y la inclusión efectiva de átomos de Mn de manera substitucional en la red de GaAs, consiguiendo películas delgadas en distintas concentraciones, lo que indica que la obtención de películas delgadas semiconductoras GaAs dopadas con Mn, por magnetrón sputtering, es factible y reproducible. A partir de difracción de rayos-x se evidenció la co-existencia de fases de GaAs y MnAs, lo cual fue confirmado los espectros Raman, donde se identificaron modos vibracionales característicos de MnAs y GaAs (TO y LO del semiconductor GaAs). La relación de intensidades de los modos TO para las muestras crecidas a $100{ }^{\circ} \mathrm{C}$ y $200{ }^{\circ} \mathrm{C}$ puede estar asociada a un reordenamiento estructural en la matriz de GaAs por la inclusión de átomos de $\mathrm{Mn}$, posiblemente de manera substitucional. A través de imágenes AFM, se determinó el valor promedio de rugosidad y tamaño de partícula en cada una de las muestras. También se observaron clusters originados probablemente en regiones ricas en Mn y con menor movilidad del Mn a una baja temperatura del sustrato. Finalmente, las imágenes de MFM permitieron constatar la formación de dominios magnéticos ligados a la presencia de aglomeraciones de MnAs, responsable del magnetismo a altas temperaturas en aleaciones semi-magnéticas de GaAsMn. 


\section{AGRADECIMENTOS}

Este trabajo fue financiado parcialmente por la dirección de investigaciones (DIMA), proyecto "Películas delgadas basados en semiconductores III-V y III-V: Mn para aplicaciones en sensores de efecto Hall". Código: 38416.

\section{BIBLIOGRAFIA}

[1] KAO, Y., CHOU, H., HSU, S.,et al., "Performance comparison of III-V//Si and III-V//InGaAs multijunction solar cells fabricated by the combination of mechanical stacking and wire bonding", Scientific Reports, v. 9, n. 1, 4308, pp. 1-11, Mar. 2019.

[2] METAFERIA, W., SCHULTE, K., SIMON, J., et al., "Gallium arsenide solar cells grown at rates exceeding $300 \mu \mathrm{m} \mathrm{h}-1$ by hydride vapor phase epitaxy", Nature Communications, v. 10, n. 1, 3361, pp. 1-8, Dec. 2019.

[3] LONG, J., XIAO, M., HUANG, X., et al., "High efficiency thin film GaInP/GaAs/InGaAs inverted metamorphic (IMM) solar cells based on electroplating process", Journal of Crystal Growth, v. 513, n. 1, pp. 38-42, May. 2019.

[4] YILDIZ, Y., BILEN, K., BOSI, M., et al., "The thermoelectric power factor enhancement of GaAs $\mathrm{G}_{1-\mathrm{x}} \mathrm{N}_{\mathrm{x}}$ ", Journal of Optoelectronics and Advanced Materials, v. 20, n. 9, pp. 515-519, May. 2018.

[5] KOSA, A., STUCHLIKOVA, L., HARMATHA, L., et al., "DLTS study of InGaAs and GaAsN structures with different indium and nitrogen compositions", Materials Science in Semiconductor Processing, v. 74, n. 1, pp. 313-318, Feb. 2018.

[6] SANDOVAL-SANTANA, J., IBARRA-SIERRA, V., AZAIZIA, S., et al., "Electron-nuclear spin dynamics of Ga centers in GaAsN dilute nitride semiconductors probed by pump-probe spectroscopy", The European Physical Journal Plus, v. 133, n. 3, 122 pp. 1-10, Mar. 2018.

[7] COLONNA, S., SESSI, V., PLACIDI, E., et al., "Two-dimensional antiferromagnetic ordering of the Mn/GaAs(001) interface", Physical Review B, v. 99, n. 11, 115311 pp. 1-8, Mar. 2019.

[8] SOLTANOVICH O., KOVALSKIY, V., VERGELES, P., et al., "Study of extended electrically active defects in heterostructures based on $(\mathrm{Ga}, \mathrm{Mn}) \mathrm{As} /(\mathrm{In}, \mathrm{Ga}) \mathrm{As}$ by electron beam-induced current and deep-level transient spectroscopy", Journal of Surface Investigation: X-ray, Synchrotron and Neutron Techniques, v. 13, n. 1, pp. 105-110, Jan. 2019.

[9] DE SANTIAGO, A., SÁNCHEZ-VALDÉS C., SÁNCHEZ LLAMAZARES, J., et al., "Magnetic properties of GaAs:Mn self-assembled nanostructures grown at relatively high-temperature by Molecular Beam Epitaxy", Journal of Magnetism and Magnetic Materials, , v. 475, n. 1, pp. 715-720, Apr. 2019.

[10] YARZHEMSKYA, V., MURASHOVA, S., IZOTOVA A., Electronic structure and ferromagnetic transition temperature of $\mathrm{Ga}_{1-\mathrm{x}} \mathrm{Mn}_{\mathrm{x}} \mathrm{As}$ in the nonempirical local exchange method, Inorganic Materials, v. $55, \mathrm{n}$. 1, pp. 1-8, Jan. 2019.

[11] KEQI, A., GEHLMANN, M., CONTI, G., et al., "Electronic structure of the dilute magnetic semiconductor $\mathrm{Ga}_{1-\mathrm{x}} \mathrm{Mn}_{\mathrm{x}} \mathrm{P}$ from hard $\mathrm{x}$-ray photoelectron spectroscopy and angle-resolved photoemission" Physical Review B, v. 97, n. 15, 155149, pp. 1-11, Aug. 2018.

[12] GAN'SHINA, E., GOLIK, L., KUN'KOVA, Z et al., "Magneto-optical spectroscopy of diluted magnetic semiconductorsGaMnAs prepared by ion implantation and further impulse laserannealing" Journal of Magnetism and Magnetic Materials, v. 459, n. 1, pp. 141-146, Aug. 2018.

[13] LIU, X., DOBROWOLSKA, M., FURDYNA, J.K., et al., "Observation of two-critical-angle phenomenon in spin wave resonances in diluted ferromagnetic semiconductor GaMnAs thin films", Journal of Magnetism and Magnetic Materials, v 494. n. 1, 165752 pp. 1-8, Jan 2020.

[14] LIU, X., SASAKI Y., FURDYNA, J.K. "Ferromagnetic resonance in $\mathrm{Ga}_{1-\mathrm{x}} \mathrm{Mn}_{\mathrm{x}}$ As: Effects of magnetic anisotropy”, Physical Review B, v. 67, n. 1, 205204 pp. 1-9, Nov. 2003.

[15] LEE, H., LEE, S., CHOI, S., et al., "Interlayer exchange coupling in MBE-grown GaMnAs-based multilayer systems", Journal of Crystal Growth, v. 477, n. 1, pp. 188-192, Nov. 2017.

[16] REYES, A., PRIETO, E., OMAMBAC, K., et al., "Terahertz emission characteristics of GaMnAs dilute magnetic semiconductor under $650 \mathrm{mT}$ external magnetic field", Current Applied Physics, v. 17, n. 4, pp. 522-526, Apr. 2017.

[17] SPIESSER, A., SATO, Y., SAITO, H., et al., "Epitaxial growth of ferromagnetic semiconductor $\mathrm{Ga}_{1-}$ 
${ }_{x} \mathrm{Mn}_{\mathrm{x}}$ As film on Ge (001) substrate", Thin Solid Films, v. 536, n. 1, pp. 323-326, Jun. 2013.

[18] XU, P., QI, D., ACKERMAN, M., et al., "Controlling Mn depth profiles in GaMnAs during hightemperature molecular beam epitaxial growth", Journal of Crystal Growth, v. 327, n. 1, pp. 42-45, Jul. 2011.

[19] LEITE, D., DA SILVA, L., PEREIRA, A., et al., "Nanocrystalline $\mathrm{Ga}_{1-\mathrm{x}} \mathrm{Mn}_{\mathrm{x}} \mathrm{N}$ films grown by reactive sputtering", Journal of Crystal Growth, v. 294, n. 2, pp. 309-314, Sep. 2006.

[20] PULZARA-MORA, A., LÓPEZ-LÓPEZ, M., ROSALES-RIVERA, A., "Vibrational raman modes relating to $\mathrm{Mn}_{\mathrm{m}} \mathrm{As}_{\mathrm{n}}$ clusters", Revista Colombiana de Física, v. 42, n. 3, pp. 457-460, Mar. 2011.

[21] YANG, J., CHEN, N., LIU, Z., et al., "(Ga,Mn,As) compounds grown on semiinsulating GaAs with mass-analyzed low energy dual ion beam deposition", Journal of Crystal Growth, v. 234, n. 2, pp. 359-363, Sep. 2002.

[22] SHERRER, P., "Bestimmung der grösse und der inneren struktur von kolloidteilchen mittels röntgensrahlen", Mathematisch-Physikalische Klasse, v. 2, n. 1, pp. 387-388, Jul. 1918.

[23] NUOFU, C., HUIXIN, X., JUNLING, Y., et al., "Content analyses in GaMnAs by double-crystal X-ray diffraction”, Chinese Science Bulletin, v. 47, n. 4, pp 274-275, Feb. 2002.

[24] MENDOZA-ÁLVAREZ, MA. E., TABARES-MUÑOZ, C., "Óxidos como sustratos para películas delgadas de superconductores de alta temperatura crítica", Revista Mexicana de Física, v. 46, n. 1, pp. 8-13, Feb. 2000.

[25] ZHANG, Y. H., GUO, L.L., SHEN, W. Z., "Study on the Raman scattering measurements of Mn ion implanted GaN", Materials Science and Engineering B, v.130, n. 1-3, pp. 269-272, Jun. 2006.

[26] LEITE ALVES, H., SCOLFARO L., DA SILVA JR, E., "Lattice dynamics of $\mathrm{Ga}_{1-\mathrm{x}} \mathrm{Mn}_{\mathrm{x}} \mathrm{N}$ and $\mathrm{Ga}_{1-\mathrm{x}} \mathrm{Mn}_{\mathrm{x}}$ As by first-principle calculations", Nanoscale Research Letters, v. 7, n. 1, pp. 1-5, Oct. 2012.

[27] TROMMER, R., HÜLLER, H., CARDONA, M., et al., "Dependence of the phonon spectrum of InP on hydrostatic pressure", Physical Review B, v. 25, n. 10, pp. 4869-4878, May. 1980.

[28] SAUNCY, T., HOLTZ, M., "Effect of pressure on a defect-related band-resonant vibrational mode in implantation disordered GaAs", Physical Review B, v. 50, n. 15, pp. 10702-10705, Oct. 1994.

[29] TECH, H., Micromagnetic investigation of MnAs thin films on GaAs surfaces, Tesis Dr. rer. nat.. Humboldt-Universitat zu, Berlin, Alemania, 2005.

\section{ORCID}

José Doria-Andrade Camilo Pulzara-Mora Roberto Bernal-Correa Andrés Rosales-Rivera Álvaro Pulzara-Mora http://orcid.org/0000-0002-8014-5589

http://orcid.org/0000-0001-9652-3225

http://orcid.org/0000-0001-9339-6574

http://orcid.org/0000-0002-8451-1304

http://orcid.org/0000-0003-1648-1788 